\title{
VIBRATION OF VISCOELASTIC BARS USING A FRACTIONAL VOIGT AND SCOTT-BLAIR MODELS
}

\author{
P. Labędzki ${ }^{*}$, R. Pawlikowski***
}

\begin{abstract}
The paper presents dynamic analysis of a bar with one end fixed and other free, loaded with force at its free end. The viscoelastic material of the bar is described by fractional Voigt or Scott-Blair models. Rayleigh-Ritz and Laplace transform methods were applied to obtain closed-form solution of the considered problem.
\end{abstract}

Keywords: viscoelasticity, fractional calculus, fractional Voigt model, Scott-Blair model

\section{Introduction}

Fractional calculus has been successfully applied to modeling of viscoelastic materials (Mainardi, 2010). Many authors considered such material models in dynamic analysis of beams, for example (Di Paola et al., 2013; Lewandowski and Wielentejczyk, 2017; Martin, 2017). In this paper we consider vibration of a bar with one end fixed and other free, loaded with force at its free end. The simplest viscoelastic materials models are used: fractional Voigt and Scott-Blair models. General theory of the nonhomogeneous fractional equations with constant coefficients and Laplace transform (see (Srivastava et al., 2006)) were applied to solve the stated problem.

The paper is organized as follows: First some definitions and notations from fractional calulus are introduced and constitutive relations between stress and strain for fractional Voigt and Blair-Scot material models are written. In section 3 we state the problem. In section 4 we solve it. In section 5 we give some numerical examples of solutions of obtained fractional differential equations.

\section{Preliminaries and notations}

There exist many definitions of fractional derivative. The most popular in application to viscoelasticity are Riemann-Liouville and Caputo fractional derivatives (Mainardi, 2010). For $0<\alpha<1$ we define them respectively as follows:

$$
\begin{aligned}
D_{\mathrm{RL}}^{\alpha} F(t) & =\frac{d}{d t}\left(\frac{1}{\Gamma(1-\alpha)} \int_{0}^{t} F(\tau)(t-\tau)^{-\alpha} d \tau\right) \\
D_{\mathrm{C}}^{\alpha} F(t) & =\frac{1}{\Gamma(1-\alpha)} \int_{0}^{t} \frac{d F(\tau)}{d \tau}(t-\tau)^{-\alpha} d \tau
\end{aligned}
$$

These definitions are not equivalent. It is well known paradox that Riemann-Liouville derivative of constant function is not equal to zero while Caputo derivative is. There are also some problems with initial conditions for fractional differential equations with Riemann-Liouville derivatives (Bagley, 2007; Heymans and

Paweł Łabędzki, PhD.: Kielce University of Technology, al. Tysiąclecia Państwa Polskiego 7, 25-314, Kielce; Poland, pawlab@tu.kielce.pl

** Rafał Pawlikowski, PhD.: Kielce University of Technology, al. Tysiąclecia Państwa Polskiego 7, 25-314, Kielce; Poland, rpawlikowski@tu.kielce.pl 
Podlubny, 2006). This is the reason why some authors consider only Caputo derivatives, however when $F\left(0^{+}\right)=0$, then (Mainardi, 2010):

$$
D_{\mathrm{RL}}^{\alpha} F(t)=D_{\mathrm{C}}^{\alpha} F(t)
$$

In this paper we consider problem with zero initial conditions, so, in this case, both Riemann-Liouville and Caputo derivatives are equivalent and further we will denote fractional derivative of order $\alpha$ as $D^{\alpha} F(t)$.

Laplace transform of $D^{\alpha} F(t)$ can be obtained in the form (Kazem, 2013):

$$
\mathcal{L}\left(D^{\alpha} F(t)\right)=s^{\alpha} \mathcal{L}(F(t))
$$

Constitutive relation between stress $\sigma(t)$ and strain $\varepsilon(t)$ for fractional Viogt model of viscoelastic material is given by the formula:

$$
\sigma(t)=E \varepsilon(t)+\eta D^{\alpha} \varepsilon(t)
$$

where $E, \eta$-material constants, $0<\alpha<1$. If we put $E=0$, we get Scott-Blair model.

\section{Problem formulation}

Let us consider bar with lenght $L$ and cross section $A$ with one end fixed and other free, loaded with force $f(t)$ at its free end, where $f(t)$ is a given time function. Dynamic equation for axial displacement $u(x, t)$ of the bar with boundary and initial conditions are written below (dots denote time derivatives, primes spatial):

$$
\begin{aligned}
\rho \ddot{u}(x, t) & =\sigma^{\prime}(x, t)+\frac{f(t) \delta(x-L)}{A} \\
u(0, t) & =0, \sigma(L, t)=0 \\
u(x, 0) & =0, \dot{u}(x, 0)=0
\end{aligned}
$$

where $\delta$ - Dirac delta. When we put formula (1) into equation (2) and use standard formula $\varepsilon=u^{\prime}(x, t)$, we obtain (fractional derivative $D^{\alpha}$ refers to time):

$$
\ddot{u}(x, t)=\frac{E}{\rho} u^{\prime \prime}(x, t)+\frac{\eta}{\rho}\left(D^{\alpha} u(x, t)\right)^{\prime \prime}+\frac{f(t) \delta(x-L)}{\rho A}
$$

\section{Solution}

We use Rayleigh-Ritz method to solve (5) with conditions (3),(4), (Preumont, 2013). We search solution of (5) in the form:

$$
u(x, t)=\sum_{n=1}^{\infty} w_{n}(t) \Phi_{n}(x)
$$

where $w_{n}(t)$ - unknown time functions, $\Phi_{n}(x)-n$-th mode of free vibration of the bar. They are known functions:

$$
\Phi_{n}(x)=\sqrt{\frac{2}{L}} \sin \left(\lambda_{n} x\right), \quad \lambda_{n}=\frac{(2 n-1) \pi}{2 L} n=1,2, \ldots
$$

They satisfy the orthogonality conditions:

$$
\int_{0}^{L} \Phi_{i}(x) \Phi_{j}(x) d x=\delta_{i j}, \quad \int_{0}^{L} \Phi_{i}^{\prime \prime}(x) \Phi_{j}(x) d x=-\lambda_{i}^{2} \delta_{i j}, \quad i, j=1,2, \ldots
$$

where $\delta_{i j}$ - Kronecker delta. Functions $\Phi_{n}$ fulfill boundary conditions (3) and consequently function (6) also.

When we substitute (6) into (5) we obtain:

$$
\sum_{n=1}^{\infty}\left(\ddot{w}_{n}(t) \Phi_{n}(x)-\frac{\eta}{\rho} \Phi_{n}^{\prime \prime}(x) D^{\alpha} w_{n}(t)-\frac{E}{\rho} \Phi_{n}(x) w_{n}(t)\right)=\frac{f(t) \delta(x-L)}{\rho A}
$$


Let us multiply last equation by $\Phi_{i}$ for some $i=1,2 \ldots$ and integrate both sides of it in interval $0 \leq x \leq L$. Using orthogonality conditions (7) we obtain equation in time domain:

$$
\ddot{w}_{i}(t)+a_{i} D^{\alpha} w_{i}(t)+b_{i} w_{i}(t)=\tilde{f}_{i}(t), \quad i=1,2, \ldots
$$

where

$$
a_{i}=\frac{\eta \lambda_{i}^{2}}{\rho}, b_{i}=\frac{E \lambda_{i}^{2}}{\rho}, \tilde{f}_{i}(t)=\frac{(-1)^{i-1} f(t)}{A \rho}
$$

Closed-form solution of (8) one obtained by using Theorem 5.5 from (Srivastava et al., 2006):

$$
w_{i}(t)=\int_{0}^{t}(t-\tau) G(t-\tau) \tilde{f}_{i}(\tau) d \tau
$$

where

$$
G(t)=\sum_{n=0}^{\infty} \sum_{k=0}^{\infty} \frac{\left(-a_{i}\right)^{k}\left(-b_{i}\right)^{n}\left(\begin{array}{c}
n+k \\
k
\end{array}\right)}{\Gamma(2 n+(2-\alpha) k+2)} t^{2 n+k(2-\alpha)}
$$

for $b_{i}=0$ (Scott-Blair model) we have:

$$
G(t)=E_{2-\alpha, 2}\left(-a_{i} t^{2-\alpha}\right)
$$

where $E_{\alpha, \beta}(t)$ - two-parameter Mittag-Leffler function defined as:

$$
E_{\alpha, \beta}(t)=\sum_{n=0}^{\infty} \frac{t^{n}}{\Gamma(n \alpha+\beta)}
$$

Using Lemma 5 from (Kazem, 2013) we can obtain $w_{i}(t)$ for force $\tilde{f}_{i}(t)=f_{0} t^{N}$, where $N=0,1, \ldots$ and $f_{0}$ - some constant, in following form:

$$
w_{i}(t)=f_{0} N ! t^{N+2} \sum_{n=0}^{\infty} \sum_{k=0}^{\infty} \frac{\left(-a_{i}\right)^{k}\left(-b_{i}\right)^{n}\left(\begin{array}{c}
n+k \\
k
\end{array}\right)}{\Gamma(k(2-\alpha)+2 n+N+3)} t^{k(2-\alpha)+2 n}
$$

\section{Results}

Here we give some numerical examples of solutions of equation (8) given by (9)-(12). Let us put $a_{i}=1$, $b_{i}=1$ (however in one example we set $b_{i}=0$ to have Scott-Blair model).
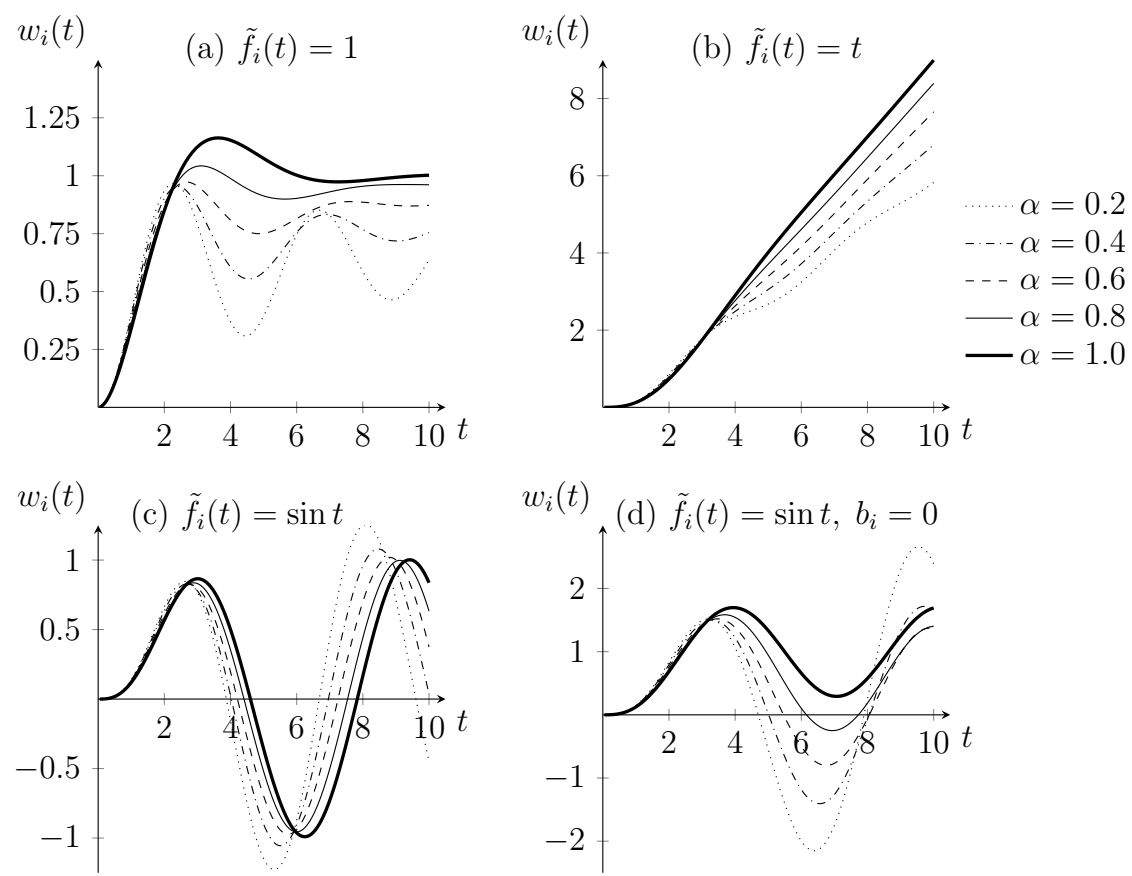

Fig. 1: Example solutions of equation (8) 
It is worth noting, that for $\alpha=1$, we have $D^{\alpha}=\frac{d}{d t}$ and in this case (8) takes the classical form:

$$
\ddot{w}_{i}(t)+a_{i} \dot{w}_{i}(t)+b_{i} w_{i}(t)=\tilde{f}_{i}(t)
$$

Using standard methods for solving of the ordinary differential equations we obtained following solutions of this equation for $\tilde{f}_{i}(t)=1, \tilde{f}_{i}(t)=t, \tilde{f}_{i}(t)=\sin t$ and $\tilde{f}_{i}(t)=\sin t, b_{i}=0$ cases respectively $\left(a_{i}=1\right.$, $b_{i}=1$ except for the last case, where $\left.b_{i}=0\right)$ :

$$
\begin{aligned}
& w_{i}(t)=1-\frac{1}{3} e^{-t / 2}\left(\sqrt{3} \sin \left(\frac{\sqrt{3} t}{2}\right)+3 \cos \left(\frac{\sqrt{3} t}{2}\right)\right) \\
& w_{i}(t)=t-\frac{1}{3} e^{-t / 2}\left(\sqrt{3} \sin \left(\frac{\sqrt{3} t}{2}\right)-3 \cos \left(\frac{\sqrt{3} t}{2}\right)\right)-1 \\
& w_{i}(t)=\frac{1}{3} e^{-t / 2}\left(\sqrt{3} \sin \left(\frac{\sqrt{3} t}{2}\right)+3 \cos \left(\frac{\sqrt{3} t}{2}\right)\right)-\cos (t) \\
& w_{i}(t)=\frac{1}{2}(-\sin (t)-\cos (t)+\sinh (t)-\cosh (t)+2)
\end{aligned}
$$

One could check that graphs of above functions are the same as these for $\alpha=1$ in Fig. 1 .

The graphs of $w_{i}(t)$ for $\tilde{f}_{i}(t)=1$ are presented in Fig. 1a. One can observe when $\alpha$ increase, vibration amplitude decrease. It can also be seen from these graphs, that $w_{i}(t)$ oscilate around some asymptotic value $b$, and this value increase with $\alpha$ (for $\alpha=1$ from solutions of classical equation: $b=1$ ). Similar conclusions can be drawn from the graphs in Fig. $1 \mathrm{~b}$ (for $\tilde{f}_{i}(t)=t$ ): $w_{i}(t)$ oscilate around asymptotic functions $w_{\text {asymptotic }}(t)=a t+b$ for some constants $a, b$, vibration amplitude decrease, when $\alpha$ increase and coefficient $a$ increase with $\alpha$ (for $\alpha=1$ one can see from solutions of classical equation that $a=1$ and $b=-1)$.

The graphs of $w_{i}(t)$ for $\tilde{f}_{i}(t)=\sin t$ are presented in Fig. 1c and in Fig. $1 \mathrm{~d}\left(b_{i}=0\right.$, Scott-Blair model). One can observe that when $\alpha$ increase, vibration amplitude decrease and its frequency increase.

\section{Conclusions}

Dynamic analysis of the bar made from viscoelastic material loaded at its free end with given force varying with time was caried out with help of Reyleigh-Ritz and Laplace transform methods. Fractional Voigt and Scott-Blair models of the viscoelastic material has been assumed. Closed-form solutions of obtained fractional differential equations has been found. Some numerical examples of the solutions of those equations has been given. It has been noted that with order of derivative decrease vibration amplitude.

\section{References}

Bagley, R. (2007) On the equivalence of the Riemann-Liouville and the Caputo fractional order derivatives in modeling of linear viscoelastic materials. Fractional Calculus and Applied Analysis, 10(2), 123-126.

Di Paola, M., Heuer, R. and Pirrotta, A. (2013) Fractional visco-elastic Euler-Bernoulli beam. International Journal of Solids and Structures, 50(22-23), 3505-3510.

Heymans, N., and Podlubny, I. (2006) Physical interpretation of initial conditions for fractional differential equations with Riemann-Liouville fractional derivatives. Rheologica Acta, 45(5), 765-771.

Kazem, S. (2013) Exact solution of some linear fractional differential equations by Laplace transform. International Journal of nonlinear science, 16(1), 3-11.

Lewandowski, R. and Wielentejczyk, P. (2017) Nonlinear vibration of viscoelastic beams described using fractional order derivatives. Journal of Sound and Vibration, 399, 228-243.

Mainardi, F. (2010) Fractional Calculus and Waves in Linear Viscoelasticity: An Introduction to Mathematical Models. World Scientific, Singapore.

Martin, O. (2017) Nonlinear dynamic analysis of viscoelastic beams using a fractional rheological model. Applied Mathematical Modelling, 43, 351-359.

Preumont, A. (2013) Twelve lectures on structural dynamics. Springer.

Srivastava, H.M., Kilbas, A.A. and Trujillo, J.J. (2006), Theory and applications of fractional differential equations, North-holland mathematics studies, vol. 204, Elsevier, Amsterdam 